Review

\title{
Developments in Contact Lens Imaging: New Applications of Optical Coherence Tomography
}

\author{
Joanna Stachura *, Małgorzata Seredyka-Burduk, Ilona Piotrowiak-Słupska, \\ Magdalena Kaszuba-Modrzejewska, Jagoda Rzeszewska-Zamiara ${ }^{\circledR}$ and Bartłomiej J. Kałużny
}

Division of Ophthalmology and Optometry, Department of Ophthalmology, Collegium Medicum, Nicolaus Copernicus University, Bydgoszcz 85-168, Poland

* Correspondence: joannastachura@icloud.com

Received: 17 May 2019; Accepted: 24 June 2019; Published: 26 June 2019

\begin{abstract}
Optical coherence tomography (OCT) is a high-speed and non-contact optical imaging technology widely used for noninvasive cross-sectional imaging of biological objects. Two main OCT technologies have been developed: time domain and Fourier domain technologies. The latter can be further divided into spectral domain OCT, which uses a broadband light source and a spectrometer as a detector, and swept source OCT, which employs a quickly-rotating laser source. Advances in OCT technology have made it one of the most helpful devices in ophthalmic practice. Fourier OCT has revolutionized imaging of the posterior segment of the eye, as well as of anterior structures and has enhanced the ability to diagnose and manage patients. It provides high-resolution information about the tear film, contact lens (CL), a qualitative and quantitative assessment of the anterior eye that is important in CL fitting, and allows possible eye surface changes while wearing CLs to be monitored. Potential swept source OCT technology applications include industrial processes of lens design and quality control. In this paper, we describe clinical applications ant outline a variety of multifunctional uses of OCT in the field of refractive error correction with CLs.
\end{abstract}

Keywords: contact lens imaging; optical coherence tomography

\section{Introduction}

OCT is a high-speed and non-contact optical imaging procedure used for noninvasive cross-sectional imaging of biological objects. OCT images use light backscattered from different layers of tissue to analyze the structures of the anterior eye [1-4]. OCT, in its time domain variant (TdOCT), was invented by Fujimoto et al. in 1991 [5]. Applying the OCT technique to imaging anterior eye structures was first described in 1994 by Izatt et al. using light with a central wavelength of $830 \mathrm{~nm}$ [6]. In 2001, Radhakrishnan et al. first revealed the modern version of anterior segment OCT (ASOCT) using 1310-nm wavelength light and a scan speed of 8 frames/s [7]. The development of Fourier domain OCT (FdOCT) resulted in better quality images and the possibility of more rapidly obtaining images of the anterior and posterior segment of the eye. The first paper reporting anterior segment imaging with spectral-domain OCT (SOCT) was published in 2002 [8]. In 2006, the first SOCT images of the cornea and its pathologies were presented [2]. The same year, high resolution two-dimensional SOCT images of a CL on the surface of the eye were reported [9]. Implementation of real-time imaging and recording allows OCT to obtain dynamic information, such as CL movement caused by blinking [10]. Luo and Jacobs suggested the need to create a guide on contact lens fitting with OCT [1].

There are two main types of OCTs: TdOCT, in which the position of the reference mirror is varied to produce cross-sectional images, and FdOCT, based on a fixed reference mirror and Fourier transformation of the spectral interferogram [11]. FdOCT technology, usually equipped with an 830-nm 
light source, works faster and identifies more details of all layers of the cornea in a limited area of tissue, whereas TdOCT systems for anterior segment imaging, which normally employ a 1300-nm light source, show all the structures in a single image $[4,5,12]$. In OCT, the axial resolution is determined by the central wavelength of a light source and is considerably impacted by the bandwidth of the system.

The latest technology, swept source OCT (SSOCT), is able to image structures with improved speed and sensitivity compared with the previous technologies. With SSOCT, a frequency swept light source and a high-speed detector are used to detect the interference signal as a function of time, instead of the spectrometer and camera employed in spectral-domain technology, as shown in Figure 1. In currently available commercial SSOCT instruments, the average wavelength is usually longer than in SOCT. Both SSOCT and SOCT detect A-scans in the Fourier domain and have a superior sensitivity compared to TdOCT. The SSOCT system has less sensitivity roll off with range, and the scanning range is thus wider than that of SOCT. High-speed imaging can help reduce motion artifacts [13]. Many applications have been described for SSOCT, including tear film evaluation, the in vivo assessment of all corneal layers, and measurements of corneal thickness and refractive corneal power to aid in refractive surgical planning [14,15]. Relatively recently, a group of scientists invented an even higher performance SSOCT that can be designed to tailor the resolution, imaging range, and axial scan repetition rate for the selected imaging application $[13,16,17]$.

Time domain OCT system

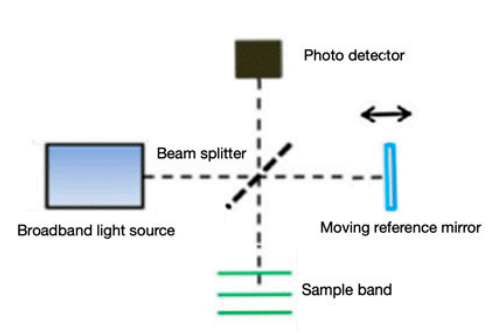

\section{Fourier domain OCT system}

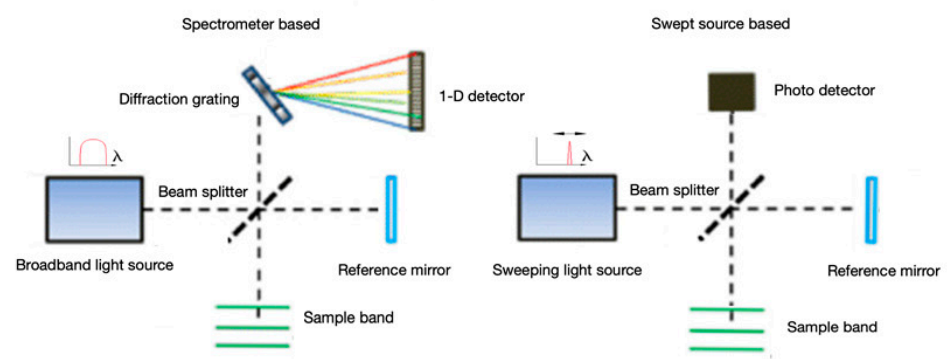

Figure 1. Schematic division of OCT systems.

En face OCT offers a new view of the different layers of tissue. It produces coronal sections (C-scans) oriented in a frontal plane, perpendicular to the optical axis of the eye. Although there are few published papers reporting the use of en face ASOCT, it was found to provide complementary information not readily available with conventional imaging techniques and new insight into different ocular surface conditions noninvasively [18].

OCT angiography (OCTA), in turn, detects blood flow by analyzing signal decorrelation or phase deviations between ultra-high-speed scans and may also be useful for examining corneal vascularization [19].

\section{Clinical Uses in CL Practice}

About $2 \%$ of the world's population wears CLs, and in developed countries, this percentage is much higher, reaching 15\% in the United States of America [20]. This number is expected to increase gradually given the various benefits that CLs provide in daily activities compared to eyeglasses. According to data from the International Survey of Contact Lens Prescribing in 2018, soft lenses accounted for about $90 \%$ of new fits and refits, and the rest used rigid gas permeable (RGP) lenses [21,22]. Scleral CLs have gained renewed interest since the late 2000s and have become an increasingly important part of RGP fittings in several countries [23].

Many factors influence lens fitting characteristics, such as the lens material and design, corneal shape, blink rate, lid-lens interactions, and post-lens tear film. Different powers, base curves, edge designs, and diameters affect the correct positioning of the CL on the eye, resulting in measurable lens lag and movement [24]. The RGP lens has a unique fitting method with "apical touch" (the lens to the 
corneal apex), intermediate clearance, and a band of touch at the junction of the optical zone and the secondary curve. The relationship between the lens edge and the cornea is another significant factor influencing optimal lens fitting and determining patient comfort and maximum wearing time [25,26]. Most studies on ocular surface shape only investigated the central cornea, which is the most important factor for vision. However, information from the peripheral part of the cornea is particularly relevant in the design and fitting assessment of CLs $[27,28]$. The general health of the patient, ergonomics, and the environment are important for the comfort of CL users.

At present, during the application of soft $\mathrm{CL}$, the practitioner routinely observes lens centration, coverage, and movement with a slit-lamp biomicroscope. Staining of the ocular tear film with fluorescein is used to evaluate the RGP contact lens fit and to assess the ocular surface after a period of wear. However, both methods provide limited information on the interaction between the lens and the ocular surface, and this examination may be insufficient to understand the cause of CL-induced discomfort and complications [28-31].

\subsection{Qualitative and Quantitative Evaluation: Surface Topography and Biometric Data}

Differences in overall lens design characteristics may play a role in user comfort, although reports are contradictory, with evidence of thicker soft lenses being both less comfortable and more comfortable than thinner lenses [32].

Shen et al. indicated that the interaction between the CL and ocular surface, especially near the edge, largely determines the comfort of the wearer and the health of the eye. They reported that a thinner edge configuration helps form a smoother transition between the edge of the lens and the conjunctiva and thus reduces mechanical irritation of the ocular surface, but may also depend on other lens factors, such as the material modulus, central thickness, base curve, and lens diameter [31].

The diameter of a soft CL is about $14 \mathrm{~mm}$, and it covers the whole cornea aligned with the corneoscleral transition zone and rests on the perilimbal sclera. In the conventional lens fitting procedure, the central corneal curvature is used to select the proper base curve of the soft CL [24]. However, the central corneal topography does not correlate with the best fitting curve for a soft CL [28]. Many researchers have assessed the corneoscleral region, reporting that, contrary to usual depictions, it is often smooth and tangential and that its topography is relevant in a CL fitting approach $[1,22,24,27,29,33]$. Hall et al. evaluated the influence of corneoscleral junction topography measured by OCT on soft CL fit and concluded that the architecture of the marginal cornea and perilimbal sclera significantly affects the optimal dynamic CL fitting [27]. Figure 2 presents a cross-sectional image of the cornea at the center (A) and the corneal center (B) and junction between the cornea and sclera $(C)$ of an eye with a soft CL obtained with high-speed SSOCT. The peripheral lens fitting has been clearly visualized.

The purposes of determining the corneal curvature profile (topography) in the initial assessments include looking for changes (particularly in terms of regularity), providing baseline data for monitoring purposes, and indicating appropriate initial fitting parameters for RGP [22].

A scleral lens requires a different fitting scheme than other lenses. Compared to corneal lenses, corneal topography is not useful as the lens rests entirely in the scleral region. Due to the knowledge of the ocular surface topography obtained from ASOCT, the corneoscleral transition shape can be described $[23,28]$. The first paper on using OCT data to design scleral lenses was published by Gemoules in 2008 [23]. Applying ASOCT, Rathi et al. measured the distance between the back surface of the scleral lens and the front surface of the cornea and reported that $90 \%$ of the eyes had a reduction in vault after four hours of lens wear compared with one hour (so one should select a trial lens with a higher than required vault) [34]. In a more recently published study on the OCT device, differences between scleral radii at the different locations (along eight meridians) were reported, and the mean scleral thickness was found to differ significantly between quadrants [35]. The measurement method discussed above may be preferred when fitting scleral lenses in the future considering the large individual differences and asymmetrical nature of the sclera. 

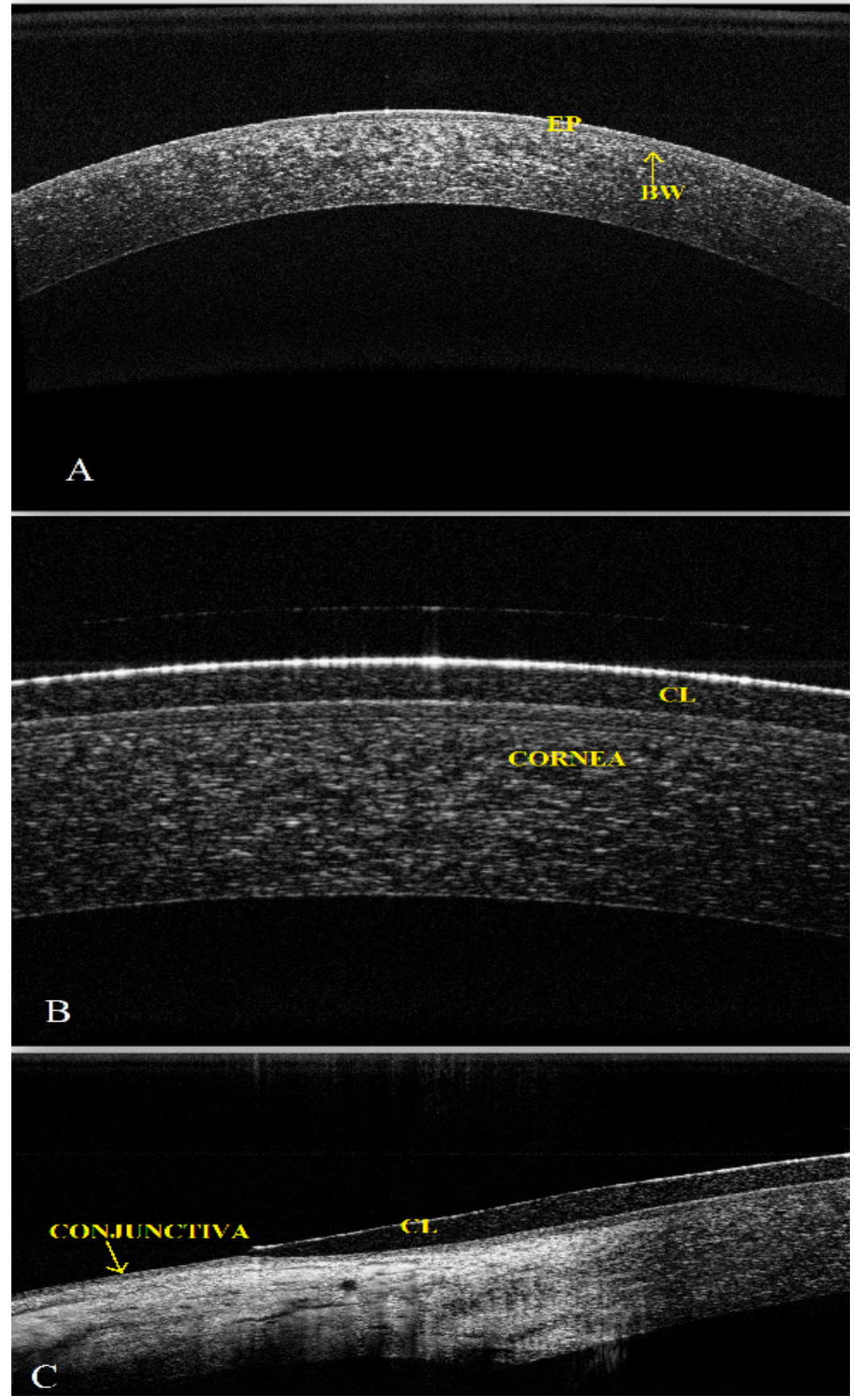

Figure 2. Optimal fitting of a soft CL. The central part of the cornea (A). The cornea with a well-fitted soft CL Dailies, Ciba Vision (B,C) (EP, epithelium; BW, Bowman membrane).

Scientific reports are lacking in the application of ASOCT in hybrid CL fitting. However, Kuczkowski et al. concluded that to ensure adequate visual acuity, it is necessary to fit a hybrid lens accurately, and ASOCT optimizes the selection of the lens [36].

Sorbara et al. showed that neither the central radius of the cornea, nor the corneal/scleral sagittal depth were highly predictive of the scleral shape. Measuring the sagittal depth directly, combining with the nasal and temporal angles when fitting very large diameter CLs may prove to be vital parameters that ASOCT can supply [37].

ASOCT is useful for the biometry of CL, cornea at the center and limbus, and sclera. It is capable of measuring pachymetry at any desired point of the cornea (visualizing the global corneal thickness map), as well as evaluating the anterior scleral thickness at specified locations. One of the major advantages 
of an OCT system is that it allows repeatable measurements of epithelial thickness and the entire corneal thickness without contact lens removal, making it an excellent device to detect in vivo corneal thickness and shape changes caused by CL wear (e.g., posterior corneal change (hypoxia)-induced corneal swelling) $[3,12,28,30,38-40]$. Examples of corneal thickness and epithelium thickness maps are shown in Figure 3 [40].

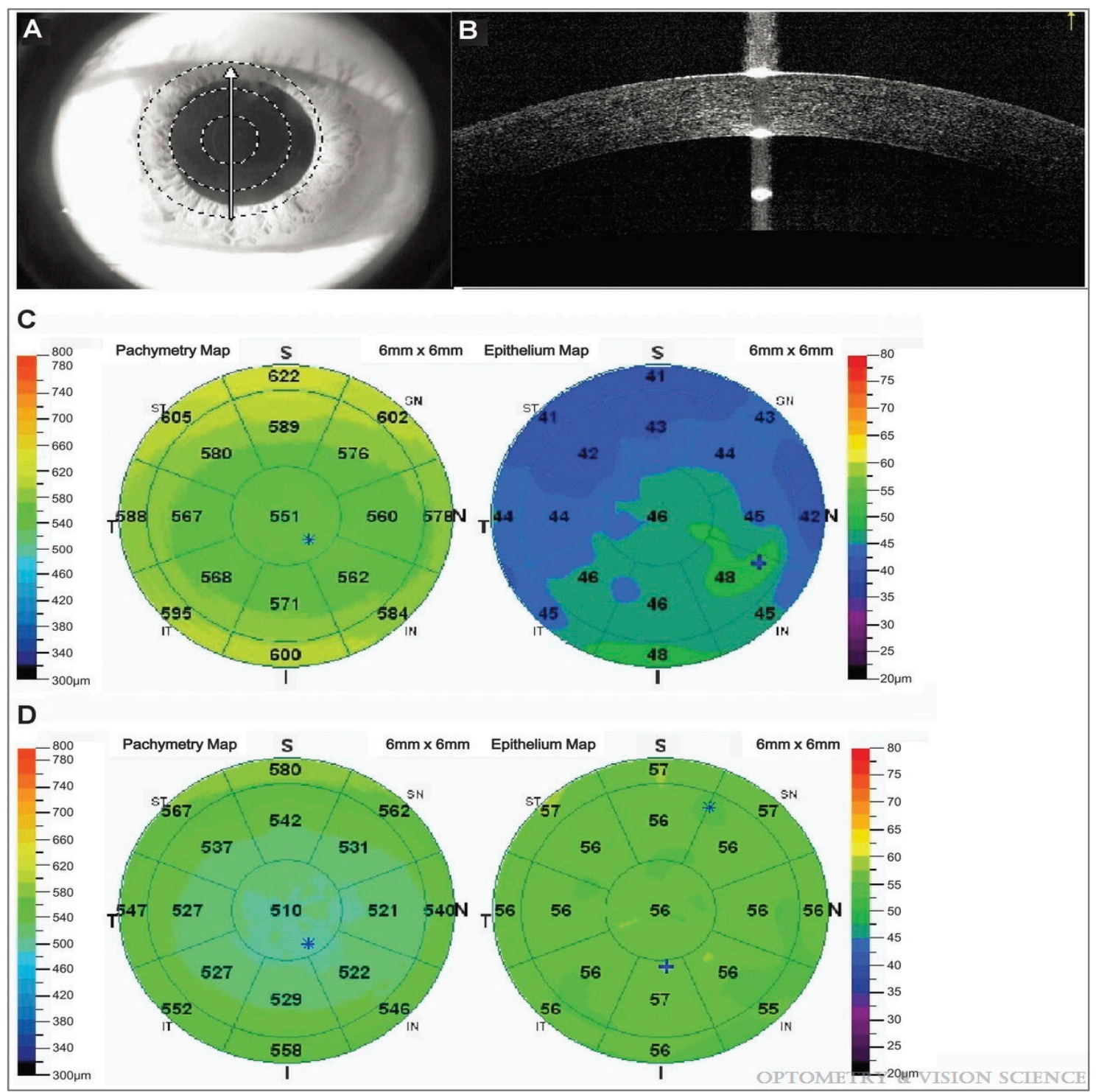

Figure 3. Central cornea's field marked for pachymetry scan (A). Cross-section projection of cornea's OCT image (B). Corneal total thickness maps and corneal epithelial thickness maps of a long-term contact lens user (C) and a non-CL wearer (D). Adapted with from Hong et al. [40].

The construction of a newer generation of OCT instruments not only provides corneal and corneal epithelial thickness mapping, but also mapping of the topography of the cornea. Maps can be obtained for both the front and back surfaces. The results of a keratometry map of the cornea are expressed in diopters or millimeters [5,12,30,41].

Karnowski et al. compared SSOCT with a Placido-based topographer and a Scheimpflug imaging-based topographer. SSOCT has some advantages over a commercial system, including the quality of tomograms and measurement time. The topographic analysis can be conducted along with high-quality cross-sectional imaging [42]. 
Doors et al. reported that central and peripheral corneal thickness measurements and pachymetry maps using ASOCT are repeatable and reproducible in healthy eyes, as well as in patients with keratoconus [5]. Li et al. presented corneal epithelial thickness mapping using SOCT, with good repeatability in both normal and keratoconic eyes [41].

\subsection{Evaluation of Tear Fluid in CL Wearers}

Tear film function is an important factor in CL use. A large proportion of CL drop-outs cite discomfort and symptoms of dryness as reasons for ceasing lens wear [43-46]. If the post-lens tear layer is diminished, as in tight lens fitting or dry eye syndrome, the accumulation of debris and dead cells may pose a significant risk to the ocular surface [25,31]. A thorough assessment of the tear film allows a more suitable lens material to be selected, such as one with a low water content (to limit dehydration) and high lubricity (to minimize friction), which are associated with a positive impact on CL comfort.

Using an older version of OCT, Wang et al. showed that it is possible to measure pre-corneal and central pre- and post-lens tear film thickness with OCT, but given the limited axial resolution of OCT, these measurements have to be indirect [47]. Later, a full-field real-time OCT with high resolution that imaged the thickness of the tear film across the vertical meridian of the ocular aperture was described. The instrument was shown to be capable of imaging and measuring the tear film thickness and upper and lower tear menisci with good repeatability (Figure 4), both with and without CL wear, although an indirect method was used to measure the central tear film thickness [16,46-51]. Using ultra-high-resolution OCT, Wang et al. proved that the thickness of the tear film under the lens edge differs with different types of soft lenses and base curves; in the case of RGP, the disparity of central tear thickness and under the edge was demonstrated [52].

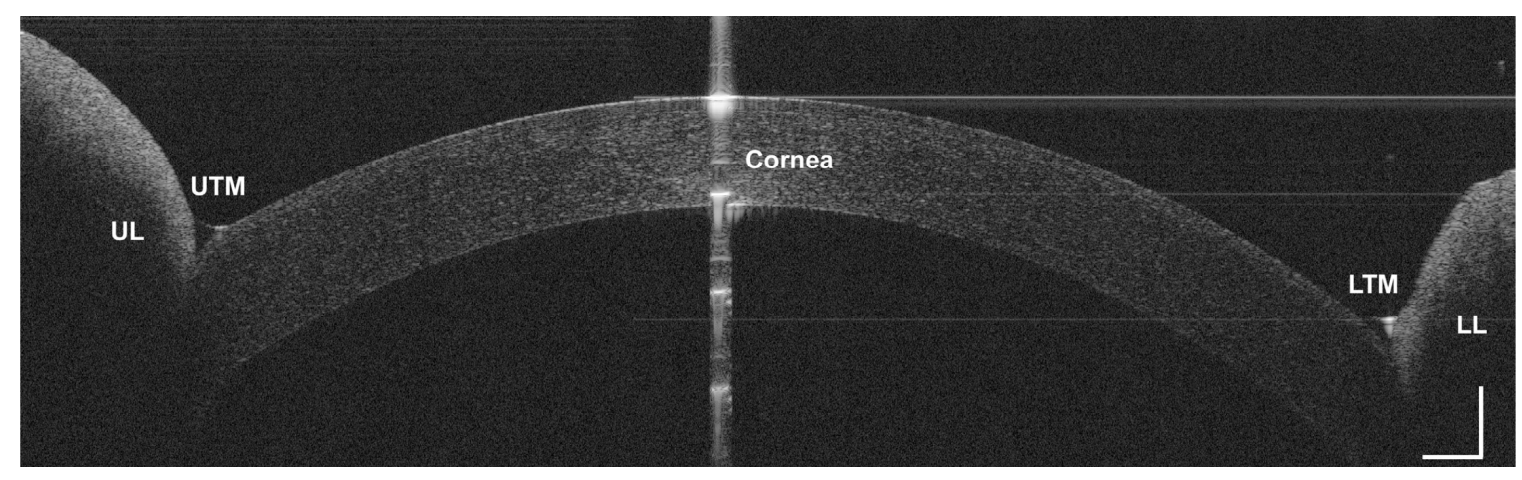

Figure 4. Analysis of tear meniscus as a cross-sectional image (LTM, lower tear meniscus; UTM, upper tear meniscus; LL, lower lid; UL, upper lid). Adapted from Tao et al. [16].

In 2003, for the first time, the thickness of the pre-lens tear film of RGP was examined. The results showed that the thickness of the pre-lens tear film on a polymethyl methacrylate lens was thicker than the thickness of the pre-corneal tear film and pre-lens tear film on soft lenses [47]. Comparatively, for the first time in 2012, OCT was used for measuring the post-lens tear film thickness in keratoconic eyes fitted with RGP lenses, without the aid of artificial tears to evaluate types of fitting patterns, either in the central fit or the variable edge lift in the examined patients [25].

The first report of the measurement of the volume of the tear meniscus using OCT was that of Fukuda et al. They demonstrated that SSOCT could measure the tear meniscus centrally in its natural state and in real time with satisfactory inter-grader and inter-image repeatability [15].

The pilot study of Tapasztó et al. examined the mechanical effect of the CL on the bulbar conjunctiva and possible changes using the lid-parallel conjunctival fold (LIPCOF) grading system with the CL on and then off, and a significant increase in the OCT-LIPCOF grading was found when the CL were fitted on the patient [43]. Shen et al. hypothesized that a higher level of conjunctival buildup around the lens edge reduced tear exchange beneath the lens and showed that OCT could 
provide an opportunity for a quick, noninvasive, and quantitative method for the assessment and follow-up of CL-related dry eye [31,32,43].

\subsection{Static and Dynamic Assessment of CL Fitting}

In the primary position, the ideal soft lens fit should be well-centered. The lens edge must always rest on the conjunctiva well clear of the limbus. A natural blink should induce movement that is observable [22,27]. This is especially important in toric and multifocal soft lens fitting. As these lenses are generally aspheric in nature, a decentered lens induces visually compromising aberrations, such as astigmatism, coma, and curvature [22]. Proper lens movement facilitates tear exchange underneath the lens and the removal of debris and inflammatory mediators [24]. Traditionally, the dynamic assessment of lens fitting has been performed via slit-lamp biomicroscopy evaluation. The ease of displacement or recovery speed of the push-up test can be registered and measured by video capture $[24,30]$. As the OCT allows for objective quantitative measurements of CL movement, it is thought to be a perfect complement to slit-lamp examination [53].

RGP lens fitting is more complicated and usually requires additional knowledge and clinical experience. The standard criterion for evaluating the lens-to-cornea fit has been to examine the fluorescein test and slit-lamp with a cobalt filter. Piotrowiak et al. compared the results of fitting RGP lenses with two methods and showed that fitting with a commercial SOCT is less accurate than the traditional subjective method according to the qualitative assessment of the difference in gaps between the front of the cornea and the lens, depending on changes in the base curve radius test lens.

In 2010, an analysis of blink-induced vertical movements of the interior lens edge of soft and rigid CLs using video-rate imaging modes of SOCT was reported. The method allowed the simultaneous real-time quantitative measurement of the movement of the CL and an objective evaluation of the fitting relationship between the lens and the ocular surface [10].

According to Cui et al., combining ultra-high-resolution and ultra-long scan-depth OCT has enabled the dynamic tracking of lens micro-movements and the overall shape of the ocular surface [24]. A recently-published study demonstrated the feasibility and good repeatability of a novel method based on the ultra-long scan-depth OCT system to assess the centration and movement characteristics of CLs [54].

\subsection{Complications}

ASOCT is a device that acquires data about different corneal structural changes, including possible complications after CL wearing. ASOCT is capable of visualizing the cornea in detail and objectively assessing the location, size, depth, shape, and light-scattering properties of the changed tissue, so it can be used to monitor the disease process. Corneal opacities or haze due to scattering, which causes an incorrect identification of the corneal surface reflections, can be precisely mapped using ASOCT, providing the corneal and epithelial thickness and the depth of the lesion $[2,4,5,9,31,55]$. All lenses need to interact with the tear film, and it is inevitable that the lens surface will have deposits. The integrity of the CL and the condition of the lens surface can also be examined using ASOCT [22].

Alonso-Caneiro et al. noticed the potential of the OCT to assess the effect of CL on the morphology of the corneoscleral transition zone [29]. The edge of the soft CL is likely to exert greater pressure in the limbal/scleral region and may cause subtle changes in the anterior layers of the cornea (especially in the perilimbal zone) that can be accurately visualized in ASOCT $[9,29,31]$. The zone of the lens with the greatest thickness difference (e.g., stabilization zones of the toric lens or the junction between the pigmented and clear regions in heavily-pigmented cosmetic soft lenses) is likely to influence the changes in morphometry [29].

The new technique, known as en face ASOCT, is capable of measuring the depth of corneal changes more precisely. Visualization in a frontal plane can reveal supplemental morphological details in patients with ocular surface conditions. This may improve the quality of diagnosis and be used to monitor the efficacy of treatments with no corneal contact [18]. 
Alabi et al. applied ultra-high-resolution OCT to image the microvascular properties within the limbus that may occur after CL wear [56]. Early pilot cross-sectional study, performed by Ang et al., suggests that the swept source OCTA used may be helpful for detecting areas of vascularization in the anterior segment [19].

\section{CL Metrology}

The authors of a few published papers have reported new applicability of OCT as a tool for the production of CLs, quality control during the CL production process, and the evaluation of new lens designs. Potentially, an SSOCT-based instrument could measure the base curve of the lenses in the production line, as well as verify the shape and surface quality [28,57-59].

In 2010, Shen et al. introduced a novel suitable research tool for imaging an entire soft CL in vitro (immersed in CL solution) and on the eye. They detected the surfaces of the lens and generated thickness maps of the soft CL (Figure 5). It was the first report using SOCT with an extended scan depth and width for directly imaging the entire CL in three dimensions (3D) [28].
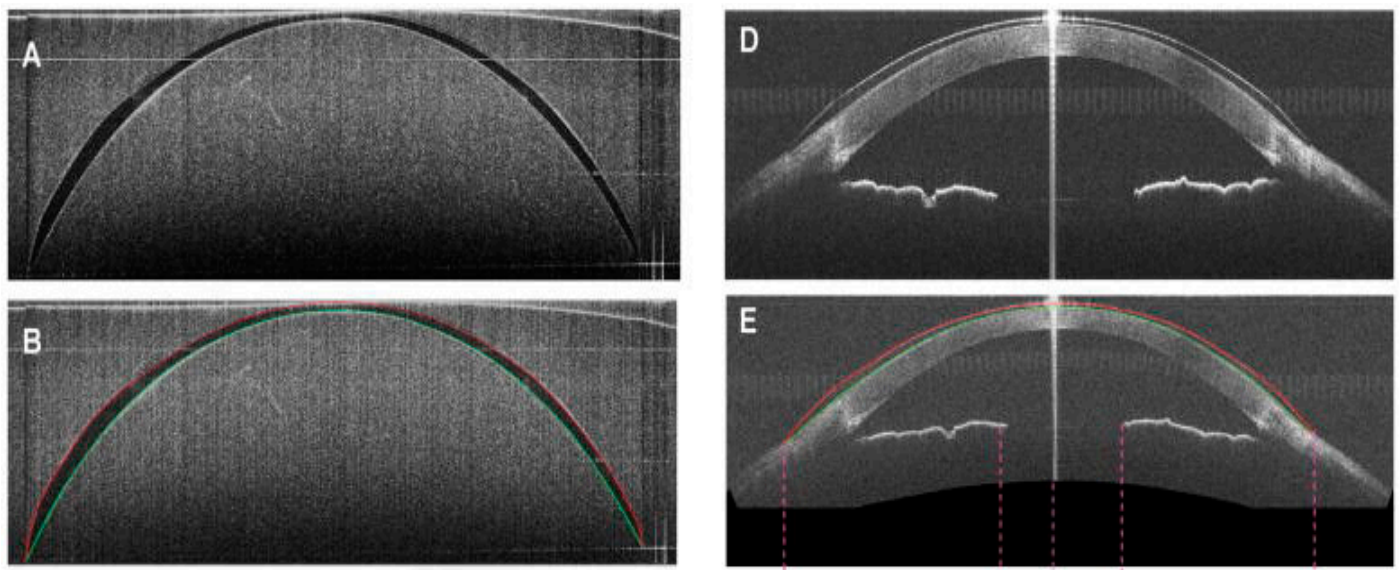

C
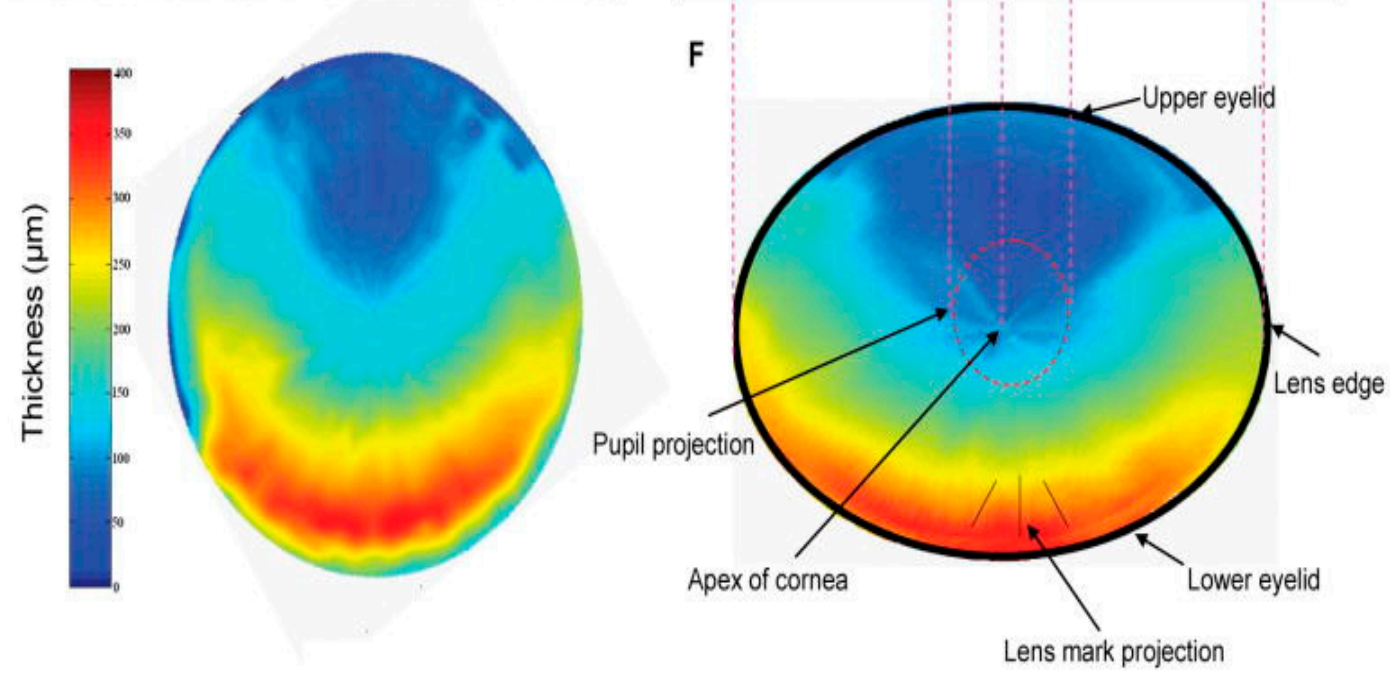

Figure 5. The whole area of CL in vitro and on the ocular surface imaged by SOCT. Cross-sectional OCT image of a toric soft CL immersed in CL solution (A) and in vivo (D) and their segmented surfaces: anterior with red color and posterior with green color can be seen in (B) and (E), respectively. Thickness map of the toric soft CL is the same in vivo (F) and in vitro (C). The resolution is $6.0 \mu \mathrm{m}$ axially and $15.0 \mu \mathrm{m}$ transversally. Adapted from Shen et al. [28].

Davidson and Barton also used OCT to measure the mechanical prism of CLs $0.3 \mathrm{~mm}$ from the edge. The prism was defined as the maximum difference in thickness at opposing points on the lens edge and considered to be a significant metric for the quality control of the CL manufacturing process. 
The thickness at this fixed distance from the edge was measured for over $360^{\circ}$. Their automated algorithm and analysis established the capability of SOCT as a tool for nondestructive, fast, and reliable CL metrology [57].

The first method to allow whole-lens, 3D metrology of CL based on SSOCT was reported by Karnowski et al. in 2014. This quantitative inspection of the lens was performed by the measurement of the samples in a dedicated wet cell with enhanced contrast by increased liquid reflectivity. An auto-positioning and auto-levelling method was developed on the basis of lateral capillary interactions between the wet cell wall and the lens floating on the liquid surface (Figure 6) [58].
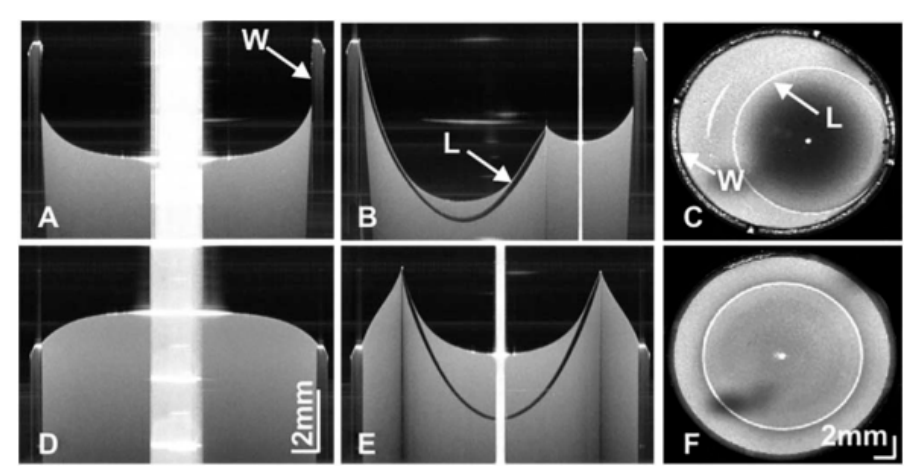

Figure 6. Experimental confirmation of the capillary forces between wet cell walls filled with a solution of increased reflectivity and a CL. Concave (A) and convex meniscus (D) formed at a partially-filled and overfilled cuvette as cross-sectional images. Cross-sections $(\mathbf{B}, \mathbf{E})$ and en face projections $(\mathbf{C}, \mathbf{F})$ showing attraction and repulsion effects (W, cuvette wall; L, contact lens). Adapted from Karnowski et al. [58].

The method allows for the visualization of the whole lens with its edges and the extraction of the information about the diameter, thickness, radius of curvature, and optical power in one simple measurement. The quantitative topographic results show good repeatability and match the manufacturers' specifications (Figure 7) [58]. The technique can be used to assess the quality of soft, rigid, and toric CLs and is free from the disadvantages of the standard methods used in lens metrology [57-59].
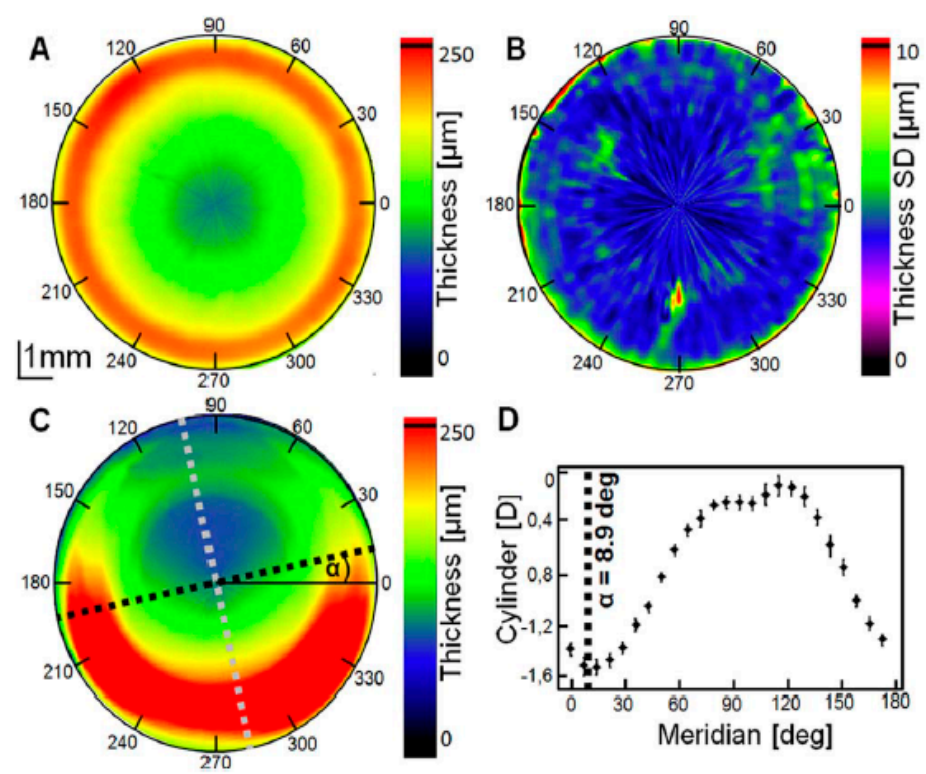

D

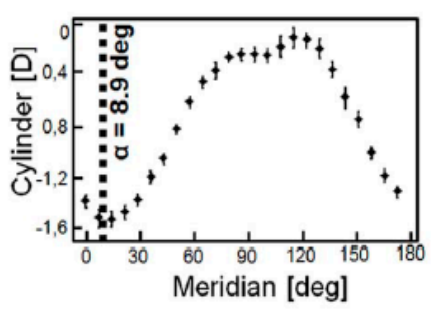

Figure 7. SSOCT of a CL's measurable estimation. Averaged thickness map (A), standard deviation map of the thickness from 10 tests of the spherical soft CL (B), and thickness of the toric soft CL shown as maps $(\mathbf{C})$; there is a brightly visible bottom weighting ballast with the axis rotated by $\alpha=8.9 \mathrm{deg}$. Astigmatism of the toric soft CL (D). Adapted from Karnowski et al. [58]. 
The capabilities of OCT (a custom-built OCT system) have been extended to provide precise thickness evaluation and high-definition 3D imaging of the edge of the soft CL immersed in different environments [60].

Coldrick et al. compared the measurement capability of an industry-focused OCT CL instrument (Optimec is830, Optimec Ltd., Malvern, United Kingdom) that is currently under development with a standard geometric inspection instrument (Optimec JCF, Optimec Ltd., Malvern, United Kingdom) that is widely used to measure the CL back optic zone radius (BOZR), central thickness, and diameter, conforming to the International Organization for Standardization requirements. The results showed that the measurements are comparable in terms of diameter, and significant improvement for BOZR and central thickness was observed with the use of Optimec is830 (Optimec Ltd., Malvern, United Kingdom). An OCT-based instrument also allowed multiple geometric parameters of CL in a single device to be investigated and provided the ability to measure geometric data, such as sagittal depth and thickness at the center and periphery of the lens. The benefits in terms of measurement time (about 35 s) strongly suggest that SSOCT may be an attractive method for measuring CLs [61].

\section{Conclusions}

OCT has a vast range of properties that are superior to traditional clinical methods. Anterior eye structures can be visualized with a high resolution, then analyzed and measured. Being a non-contact procedure, it does not influence patient comfort, permitting in vivo imaging and biometric measurement. OCT is a valuable new technology enabling corneal topography and tomography to be performed. Using OCT, we can assess the design, shape, and lens edge position and measure the scleral toricity, corneal vault, thickness of the lens, corneal epithelium, and stroma, as well as the space between the lens and the eye surface, the tear film, and other parameters that allow a faster and more precise fit, which helps the CL work better and improves the comfort of the wearer. OCT can also be useful for the diagnosis, evaluation, and documentation of CL complications and then for measuring the effectiveness of the therapy in clinical trials. Therefore, the OCT, as a powerful imaging modality, could become a standard technology applied in CL practice in the near future. Potential applications of SSOCT include industrial processes of lens design and research. Nondestructive, fast and reliable CL metrology may provide an efficient and improved quality control during and after manufacturing and may be useful in developing new materials and designs of CLs, especially in the face of the incipient revolution in contactology, including concepts such as drug-eluting lenses or ones containing electronic components.

Author Contributions: Conceptualization, B.J.K.; analysis of the literature, J.S., M.S.-B., I.P.-S., M.K.-M., J.R.-Z. and B.J.K.; writing, original draft preparation, J.S.; writing, review and editing, M.S.-B., I.P.-S., M.K.-M., J.R.-Z. and B.J.K.

Funding: This work was supported by the grant 2015/18/E/NZ5/00697 funded by the National Science Centre, Poland.

Conflicts of Interest: The authors declare no conflict of interest.

\section{References}

1. Luo,Z.K.; Jacobs, D.S. Current and Potential Applications of Anterior Segment Optical Coherence Tomography in Contact Lens Fitting. Semin. Ophthalmol. 2012, 27, 133-137. [CrossRef] [PubMed]

2. Kałużny, B.J.; Kałużny, J.J.; Szkulmowska, A.; Górczynska, I.; Szkulmowski, M.; Bajraszewski, T.; Wojtkowski, M.; Targowski, P. Spectral Optical Coherence Tomography A Novel Technique for Cornea Imaging. Cornea 2006, 25, 960-965. [CrossRef] [PubMed]

3. Wang, J.; Fonn, D.; Simpson, T. Topographical Thickness of the Epithelium and Total Cornea after Hydrogel and PMMA Contact Lens Wear with Eye Closure. Investig. Ophthalmol. Vis. Sci. 2003, 44, 1070-1074. [CrossRef] 
4. Pang, C.E.M.V.; Tan, D.T.H.; Mehta, J.S. Evaluation of Corneal Epithelial Healing Under Contact Lens Edge Fitting with Spectral-Domain Anterior Segment Optical Coherence Tomography (SD-OCT). Open Ophthalmol. J. 2011, 5, 51-54.

5. Doors, M.; Berendschot, T.T.J.M.; de Brabander, J.; Webers, C.A.B.; Nuijts, R.M.M.A. Value of optical coherence tomography for anterior segment surgery. J. Ctataract Refract. Surg. 2010, 36, 1213-1229. [CrossRef]

6. Izatt, J.A.; Hee, M.R.; Swanson, E.A.; Lin, C.P.; Huang, D.; Schuman, J.S.; Puliafito, C.A.; Fujimoto, J.G. Micrometer-scale resolution imaging of the anterior eye in vivo with optical coherence tomography. Arch. Ophthalmol. 1994, 112, 1584-1589. [CrossRef] [PubMed]

7. Radhakrishnan, S.; Rollins, A.M.; Roth, J.E.; Yazdanfar, S.; Westphal, V.; Bardenstein, D.S.; Izatt, J.A. Real-time optical coherence tomography of the anterior segment at $1310 \mathrm{~nm}$. Arch. Ophthalmol. 2001, 119, 1179-1185. [CrossRef]

8. Kałużny, J.J.; Wojtkowski, M.; Kowalczyk, A. Imaging of the anterior segment of the eye by Spectral Optical Coherence Tomography. Opt. Appl. 2002, 32, 581-589.

9. Kałużny, B.J.; Kałużny, J.J.; Szkulmowska, A.; Górczynska, I.; Szkulmowski, M.; Bajraszewski, T.; Targowski, P.; Kowalczyk, A. Spectral optical coherence tomography: A new imaging technique in contact lens practice. Ophthalmic Physiol. Opt. 2006, 26, 127-132. [CrossRef] [PubMed]

10. Kałużny, B.J.; Fojt, W.; Szkulmowska, A.; Bajraszewski, T.; Wojtkowski, M.; Kowalczyk, A. Spectral Optical Coherence Tomography in Video-Rate and 3D Imaging of contact Lens Wear. Optom. Vis. Sci. 2007, 84, 1104-1109. [CrossRef]

11. Wojtkowski, M. High-speed optical coherence tomography: Basics and applications. Appl. Opt. 2010, 49, D30-D61. [CrossRef]

12. Rio-Cristobal, A.; Marin, R. Corneal assessment technologies: Current status. Surv. Ophthalmol. 2014, 30, 599-614. [CrossRef] [PubMed]

13. Grulkowski, I.; Liu, J.J.; Potsaid, B.; Jayaraman, V.; Lu, C.D.; Jiang, J.; Cable, A.E.; Duker, J.S.; Fujimoto, J.G. Retinal, anterior segment and full eye imaging using ultrahigh speed swept source OCT with vertical-cavity surface emitting lasers. Biomed. Opt. Express 2012, 3, 2733-2751. [CrossRef]

14. Englander, M.; Xu, D.; Kaiser, P.K. Optical Coherence Tomography. In Ophtalmology, 4th ed.; Yanoff, M., Duker, J.S., Eds.; Elsevier Saunders: Philadelphia, PA, USA; Springer: Berlin/Heidelberg, Germany, 2014; pp. 448-457.

15. Fukuda, R.; Usui, T.; Miyai, T.; Yamagami, S.; Amano, S. Tear Meniscus Evaluation by Anterior Segment Swept-Source Optical Coherence Tomography. Am. J. Ophthalmol. 2013, 155, 620-624. [CrossRef] [PubMed]

16. Tao, A.; Shao, Y.; Zhong, J.; Jiang, H.; Shen, M.; Wang, J. Versatile optical coherence tomography for imaging the human eye. Biomed. Opt. Express 2013, 4, 1031-1044. [CrossRef]

17. Wojtkowski, M.; Kałużny, B.; Zawadzki, R.J. New Directions in Ophthalmic Optical Coherence Tomography. Optom. Vis. Sci. 2012, 89, 524-542. [CrossRef]

18. Hassani, R.T.J.; Liang, H.; El Sanharawi, M.; Brasnu, E.; Kallel, S.; Labbe, A.; Baudouin, C. En-face Optical Coherence Tomography as a Novel Tool for Exploring the Ocular Surface: A Pilot Comparative Study to Conventional B-Scans and in Vivo Confocal Microscopy. Ocul. Surf. 2014, 12, 285-306. [CrossRef]

19. Ang, M.; Cai, Y.; Tan, A.C.S. Swept Source Optical Coherence Tomography Angiography for Contact Lens-Related Corneal Vascularization. J. Ophthalmol. 2016, 2016, 9685297. [CrossRef] [PubMed]

20. Nichols, J.J.; Fischer, D. Contact lenses 2018. Contact Lens Spectr. 2019, 34, 18-23.

21. Morgan, P.B.; Woods, C.A.; Tranoudis, I.G.; Efron, N.; Jones, L.; Aighamdi, W.; Awasthi, S.; Belousov, V.; Bendoriene, J.; Chandrinos, A.; et al. International Contact Lens Prescribing in 2018. Contact Lens Spectr. 2019, 33, 26-32.

22. Chisholm, C.; Woods, C.A. Contact Lens Assessment. In Clinical Procedures in Primary Eye Care, 4th ed.; Elliott, D.B., Ed.; Elsevier Saunders: Cambridge, UK, 2014; pp. 112-146.

23. Van der Worp, E.; Bornman, D.; Lopes Ferreira, D.; Faria-Ribeiro, M.; Garcia-Porta, N.; González-Meijome, J.M. Modern scleral contact lens: A review. Cont. Lens Anterior Eye 2014, 37, 240-250. [CrossRef] [PubMed]

24. Cui, L.; Shen, M.; Wang, M.R.; Wang, J. Micrometer-Scale Contact Lens Movements Imaged by Ultrahigh-resolution Optical Coherence Tomography. Am. J. Ophthalmol. 2012, 153, 275-283. [CrossRef] [PubMed]

25. Elbendary, A.M.; Samra, W.A. Evaluation of rigid gas permeable lens fitting in keratoconic patients with optical coherence tomography. Graefes Arch. Clin. Exp. Ophthalmol. 2013, 251, 1565-1570. [CrossRef] 
26. Ramdas, W.D.; Vervaet, C.J.W.C.; Bleyen, I. Corneal topography for pancorneal toric edge rigid gas-permeable contact lens fitting in patients with keratoconus, and differences in age and gender. Cont. Lens Anterior Eye 2014, 37, 20-25. [CrossRef]

27. Hall, L.A.; Young, G.; Wolffsohn, J.S.; Riley, C. The Influence of Corneoscleral Topography on Soft Contact Lens Fit. Investig. Ophthalmol. Vis. Sci. 2011, 52, 6801-6806. [CrossRef] [PubMed]

28. Shen, M.; Wang, M.R.; Wang, J.; Yuan, Y.; Chen, F. Entire contact lens imaged in vivo and in vitro with spectral domain optical coherence tomography. Eye Contact Lens 2010, 36, 73-76. [CrossRef] [PubMed]

29. Alonso-Caneiro, D.; Shaw, A.J.; Collins, M.J. Using Optical Coherence Tomography to Assess Corneoscleral Morphology After Soft Contact Lens Wear. Optom. Vis. Sci. 2012, 89, 1619-1626. [CrossRef]

30. Tao, A.; Shao, Y.; Jiang, H.; Ye, Y.; Lu, F.; Shen, M.; Zhu, D.; Wang, J. Entire thickness profiles of the epithelium and contact lens in vivo imaged with high speed and high resolution optical coherence tomography. Eye Contact Lens 2013, 39, 329-334. [CrossRef]

31. Shen, M.; Cui, L.; Riley, C.; Wang, M.R.; Wang, J. Characterization of Soft Contact Lens Edge Fitting Using Ultra-High Resolution and Ultra-Long Scan Depth Optical Coherence Tomography. Investig. Ophthalmol. Vis. Sci. 2011, 52, 4091-4097. [CrossRef]

32. Morgan, P.B. Optimising contact lens wear for a lifetime of use. Optician 2013, 5, 33-37.

33. Góra, M.; Karnowski, K.; Szkulmowski, M.; Kałużny, B.J.; Huber, R.; Kowalczyk, A.; Wojtkowski, M. Ultra high-speed swept source OCT imaging of the anterior segment of human eye at $200 \mathrm{kHz}$ with adjustable imaging range. Opt. Express 2009, 17, 14880-14894. [CrossRef] [PubMed]

34. Rathi, V.M.; Mandathara, P.S.; Dumpati, S.; Sangwan, V.S. Change in vault during scleral lens trials assessed with anterior segment optical coherence tomography. Cont. Lens Anterior Eye 2017, 40, 157-161. [CrossRef]

35. Bandlitz, S.; Baumer, J.; Conrad, U.; Wolffsohn, J. Scleral topography analysed by optical coherence tomography. Cont. Lens Anterior Eye 2017, 40, 242-247. [CrossRef] [PubMed]

36. Kuczkowski, S.; Filipek, E.; Borkowska-Kuczkowska, A.; Barchanowska, D. Optical coherent tomography use in hybrid contact lenses application in keratoconus management in patients with RGP lenses intolerance-Case report. Okulistyka 2013, 4, 23-26.

37. Sorbara, L.; Maram, J.; Fonn, D.; Woods, C.; Simpson, T. Metrics of the normal cornea: Anterior segment imaging with the Visante OCT. Clin. Exp. Optom. 2010, 93, 150-156. [CrossRef]

38. Wang, J.; Simpson, T.; Fonn, D. Objective Measurements of Corneal Light-Backscatter during Corneal Swelling, by Optical Coherence Tomography. Investig. Ophthalmol. Vis. Sci. 2004, 45, 3493-3498. [CrossRef]

39. Martin, R.; Izquierdo, M.; Saber, A. Investigation of posterior corneal curvature in CL-induced corneal swelling. Cont. Lens Anterior Eye 2009, 32, 288-293. [CrossRef] [PubMed]

40. Hong, J.; Qian, T.; Yang, Y.; Jiang, C.; Sun, X.; Deng, S.X.; Xu, J. Corneal Epithelial Thickness Map in Long-Term Soft Contact Lenses Wearers. Optom. Vis. Sci. 2014, 91, 1455-1461. [CrossRef] [PubMed]

41. Li, Y.; Tan, O.; Brass, R.; Weiss, J.L.; Huang, D. Corneal Epithelial Thickness Mapping by Fourier-Domain Optical Coherence Tomography in Normal and Keratoconic Eyes. Ophthalmology 2012, 119, 2425-2433. [CrossRef]

42. Karnowski, K.; Kałużny, B.J.; Szkulmowski, M.; Góra, M.; Wojtkowski, M. Corneal topography with high-speed swept source OCT in clinical examination. Biomed. Opt. Express 2011, 2, 2709-2720. [CrossRef]

43. Tapasztó, B.; Veres, A.; Kosina-Hagyó, K.; Somfai, G.M.; Németh, J. OCT Imaging of Lid-Parallel Conjunctival Folds in Soft Contact Lens Wearers. Optom. Vis. Sci. 2011, 88, 1206-1213.

44. Evans, K.; Pult, H. How important are surface properties for successful contact lens wear? Optician 2012, 5, 14-18.

45. Chen, Q.; Wang, J.; Shen, M.; Cai, C.; Li, J.; Cui, L.; Qu, J.; Lu, F. Lower Volumes of Tear Menisci in Contact Lens Wearers with Dry Eye Symptoms. Investig. Ophthalmol. Vis. Sci. 2009, 50, 3159-3163. [CrossRef]

46. Chen, Q.; Wang, J.; Shen, M.; Cui, L.; Cai, C.; Li, M.; Li, K.; Lu, F. Tear Menisci and Ocular Discomfort during Daily Contact Lens Wear in Symptomatic Wearers. Investig. Ophthalmol. Vis. Sci. 2011, 52, 2175-2180. [CrossRef] [PubMed]

47. Wang, J.; Fonn, D.; Simpson, T.; Jones, L. Precorneal and Pre- and Postlens Tear Film Thickness Measured Indirectly with Optical Coherence Tomography. Investig. Ophthalmol. Vis. Sci. 2003, 44, 2524-2528. [CrossRef]

48. Wang, J.; Aquavella, J.; Palakuru, J.; Chung, S. Repeated measurements of dynamic tear distribution on the ocular surface after instillation of artificial tears. Investig. Ophthalmol. Vis. Sci. 2006, 47, 3325-3329. [CrossRef] 
49. Wang, J.; Aquavella, J.; Palakuru, J.; Chung, S.; Feng, C. Relationships between central tear film thickness and tear menisci of the upper and lower eyelids. Investig. Ophthalmol. Vis. Sci. 2006, 47, 4349-4355. [CrossRef] [PubMed]

50. Wang, J.; Cox, I.; Reindel, W.T. Upper and Lower Tear Menisci on Contact Lens. Investig. Ophthalmol. Vis. Sci. 2009, 50, 1106-1111. [CrossRef] [PubMed]

51. Chen, Q.; Wang, J.; Tao, A.; Shen, M.; Jiao, S.; Lu, F. Ultrahigh-Resolution Measurement by Optical Coherence Tomography of Dynamic Tear Film Changes on Contact Lenses. Investig. Ophthalmol. Vis. Sci. 2010, 51, 1988-1993. [CrossRef]

52. Wang, J.; Jiao, S.; Ruggeri, M.; Abou Shousha, M.; Chen, Q. In situ visualization of tears on contact lens using ultra high resolution optical coherence tomography. Eye Contact Lens 2009, 2, 44-49. [CrossRef] [PubMed]

53. Piotrowiak, I.; Kałużny, B.J.; Danek, B.; Chwiędacz, A.; Sikorski, B.Ł.; Malukiewicz, G. Spectral Optical Coherence Tomography vs. Fluorescein pattern for rigid gas-permeable lens fit. Med. Sci. Mon. 2014, 20, 1137-1141.

54. Cui, L.; Ming, L.; Shen, M.; Lu, F.; Wang, J. Characterization of Soft Contact Lens Fitting Using Ultra-Long Scan Depth Optical Coherence Tomography. J. Ophthalmol. 2017, 2017. [CrossRef] [PubMed]

55. Nixon, A.D.; Barr, J.T.; VanNasdale, D.A. Corneal epithelial bullae after short-term wear of small diameter scleral lenses. Cont. Lens Anterior Eye 2017, 40, 116-126. [CrossRef]

56. Alabi, E.; Hutchings, N.; Bizheva, K.; Simpson, T. Relationship between vessel diameter and depth measurements within the limbus using ultra-high resolution optical coherence tomography. J. Optom. 2018, 11, 57-65. [CrossRef]

57. Davidson, B.R.; Barton, J.K. Application of optical coherence tomography to automated contact lens metrology. J. Biomed. Opt. 2010, 15. [CrossRef]

58. Karnowski, K.; Grulkowski, I.; Mohan, N.; Cox, I.; Wojtkowski, M. Quantitative optical inspection of contact lenses immersed in wet cell using swept source OCT. Opt. Lett. 2014, 39, 4727-4730. [CrossRef] [PubMed]

59. Plainis, S.; Atchison, D.A.; Charman, W.N. Power Profiles of Multifocal Contact Lenses and Their Interpretation. Optom. Vis. Sci. 2013, 90, 1066-1077. [CrossRef] [PubMed]

60. Tankam, P.; Won, J.; Canavesi, C.; Cox, I.; Rolland, J.P. Optical assessment of soft Contact Lens Edge-Thickness. Optom. Vis. Sci. 2016, 93, 987-996. [CrossRef]

61. Coldrick, B.J.; Richards, C.; Sugden, K.; Wolffsohn, J.S.; Drew, T.E. Developments in contact lens measurements: A comparative study of industry standard geometric inspection and optical coherence tomography. Cont. Lens Anterior Eye 2016, 39, 270-276. [CrossRef] [PubMed] 